\title{
Interaction of Isentropic Compression Waves with a Bow Shock
}

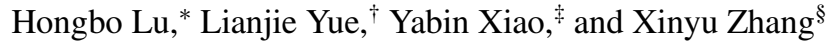 \\ Chinese Academy of Sciences, 100190 Beijing, People's Republic of China
}

DOI: $\underline{10.2514 / 1 . J 052373}$

\begin{abstract}
The interaction of isentropic compression waves with a bow shock is numerically investigated. The relative intersection region size is introduced to characterize the convergence degree of isentropic compression waves. Among the multiple parameters involved, relative intersection region size is a crucial factor in determining the occurrence of the interference patterns, as well as the corresponding pressure and thermal loads. There exist two critical relative intersection region sizes: the small one corresponds to the intersection region size being approximately equal to the transmitted shock length in the case of Edney type IV interference, and the large one corresponds to the intersection region size being close to the transmitted shock length in the case of Edney type III interference. When relative intersection region size is lower than the small critical value, Edney interference patterns take place but their types depend on the vertical intersection location. When relative intersection region size is greater than the large critical value, none of the Edney interference patterns occurs. When relative intersection region size falls in between the two critical values, part of Edney interference patterns occur and a subsonic jet is produced by the intersection of isentropic compression waves with the strong portion of the bow shock. The maximum interference heating reduces with relative intersection region size. Additionally, a dimensionless dependence of the maximum heat transfer intensification was inferred for practical design and calculations.
\end{abstract}

\section{Nomenclature}

$h \quad=$ specific enthalpy

$L_{A B}=$ strong portion length of the undisturbed bow shock

$L_{S^{\prime} E^{\prime}}=$ specific intersection region size

$M a_{0}=$ undisturbed flow Mach number

$P \quad=$ static pressure

$P_{\mathrm{st}}=$ stagnation-point pressure at the freestream conditions

$\mathrm{Pr}=$ Prandtl number

$q \quad=$ wall heat flux

$q_{\mathrm{st}}=$ stagnation-point heat flux at the freestream conditions

$\tilde{q}=$ maximum heat-transfer intensification

$R \quad=$ cylinder radius

$R_{P} \quad=$ radius of curvature of the undisturbed bow shock at the vertex $P$

$R e_{R}=$ Reynolds number based on the cylinder radius

$T_{w}=294.44 \mathrm{~K}$, wall temperature

$x, y=$ Cartesian coordinates of the undisturbed bowshock shape

$y_{C V}=$ vertical intersection location

$\beta=$ shock angle

$\Delta \quad=$ standoff distance of the undisturbed bow shock

$\delta \quad=$ flow deflection angle

$\theta=$ angular coordinate

$\mu \quad=$ Mach angle, viscosity

$\rho \quad=$ density
Received 7 October 2012; revision received 27 December 2012; accepted for publication 24 February 2013; published online 10 July 2013. Copyright (C) 2013 by the American Institute of Aeronautics and Astronautics, Inc. All rights reserved. Copies of this paper may be made for personal or internal use, on condition that the copier pay the $\$ 10.00$ per-copy fee to the Copyright Clearance Center, Inc., 222 Rosewood Drive, Danvers, MA 01923; include the code 1533-385X/13 and $\$ 10.00$ in correspondence with the CCC.

*Ph.D. Student, Institute of Mechanics, State Key Laboratory of High Temperature Gas Dynamics, Number 15 Beisihuanxi Road.

${ }^{\dagger}$ Associate Professor, Institute of Mechanics, State Key Laboratory of High Temperature Gas Dynamics, Number 15 Beisihuanxi Road; yuelj@imech.ac .cn (Corresponding Author).

*Research Assistant, Institute of Mechanics, State Key Laboratory of High Temperature Gas Dynamics, Number 15 Beisihuanxi Road.

${ }^{\S}$ Professor, Institute of Mechanics, State Key Laboratory of High Temperature Gas Dynamics, Number 15 Beisihuanxi Road.

\section{Introduction}

INCE the 1940s, achieving hypersonic flight has been a focus of $\checkmark$ research in industry, academia, and government laboratories around the world. Some hypersonic applications such as conventional reentry vehicles are generally deemed mature in terms of the established practices and the existent techniques relevant to operational vehicles. However, there remain significant challenges associated with hypersonics in light of both the performance and the operability of existing and proposed hypersonic systems such as the $\mathrm{X}-43 / 51$ and the National Aero-Space Plane (NASP) [1,2]. A fundamental design challenge of interest for high-speed vehicles is the inherent aerodynamic heating, especially that produced by shockon-shock interactions [1-ㅛ] . During hypersonic flight, shock-onshock interactions are common in many aerodynamic configurations such as launchers or propulsion systems. These shock interactions can cause extremely high localized pressure and heat-transfer rates on the vehicle surface. These features can alter the aerodynamic characteristics of the vehicle and even lead to catastrophic failures. A dramatic example of this effect was the airframe damage of the X$15 \mathrm{~A}-2$ caused by shock interactions [7,8]. Another example from the previous NASP is that the shock-shock heat flux on the scramjet engine's cowl leading edge was estimated by computational fluid dynamics (CFD) in [9] to be as much as $5.67 \times 10^{8} \mathrm{~W} / \mathrm{m}^{2}$.

Shock-wave interference is one of the most challenging problems of gas dynamics and has gained widespread interest [3, $\underline{6}, \underline{10}-\underline{20}]$. Edney [3] formulated a detailed framework for the entire spectrum of interference patterns and characterized six types of shockinterference patterns (types I-VI) depending on the strength of the impinging shock and the location of the intersecting point relative to the curved bow shock. Some refinements to these classifications have been developed subsequently, but the basic six patterns still demonstrate the range of phenomena observed, where types I, II, and $\mathrm{V}$ result in shock-wave-boundary-layer interactions, type VI involves expansion-fan-boundary-layer interactions, type III results in shear-layer impingement, and type IV is characterized by supersonic jet grazing and impingement. These shock-interference patterns generally produce a significant increase in pressure and heattransfer rate and lead to extremely high pressure and heat-transfer rate gradients in highly localized regions where the shock-interference pattern impinges on the surface. The extreme heat-transfer rate gradient leads to a large temperature gradient and attendant thermal stresses [10], which significantly reduce the service life of the materials. A combination of analytical and experimental investigations was conducted by Keyes and Hains []] to predict the pressure and heat transfer resulting from shock interaction using the 
oblique shock and Prandtl-Meyer (P-M) relationships. The results showed that both the peak pressure and the heat-transfer rate were sensitive to the upstream thermodynamic flow conditions, Mach number, and geometry. Craig and Ortwerth [11] and Wieting and Holden [12] expementally analyzed the influences of Reynolds number, Mach number, and incident shock strength. Borovoy et al. [13] examined the influence of the location of the impinging shock on the surface heat transfer through a combined computational and experimental study of type III and IV interactions. Sanderson et al. [14] extended the experimental and analytical studies on the influence of nonequilibrium dissociation on the type IV interaction and correlated the peak heat-transfer rate by a function of both freestream Mach number and flow deflection angle of the impinging shock. Lind [15], Berry and Nowak [16], and Wang et al. [17] studied the effect of body geometry on the interaction of an oblique shock with a bow shock. Lind and Lewis [18,19] and Zhong [20] studied characteristics of the type IV interference with various numerical schemes. These researches suggest that type IV interference is unsteady and produces the most serious pressure and heat transfer, which increase with Mach number and impinging shock strength and reduce with specific heat ratio.

Recently, type IV interference heating control by energy deposition and magnetogasdynamic force has drawn much attention among research communities. A series of numerical and experimental investigations by Adelgren et al. [21], Kogan and Starodubtsev [22], Kandala and Candler [23], Trumble and Candler [24], and Yan and Gaitonde $[25,26]$ were performed to examine the effectiveness of energy deposition in improving shock structure and thermomechanic loading caused by the type IV interaction. Their work demonstrated that the energy deposition diminished effectively the instantaneous peak pressure and heat fluxes. The electromagnetic forces were also introduced by Gaitonde and Miller [27] to lower heat loads on the surface under both electrodeless and electrode-based conditions. However, these active control measures add complexity to the overall system and augment the vehicle weight. Unfortunately, the hypersonic cruisers and accelerators (particularly airbreathing vehicles) are born with low thrust-minus-drag performance margins across the operating envelopes of such vehicles [28]. Consequently, some passive control methods such as aerodynamic surface configurations have to be introduced to improve the performance and feasibility of hypersonic vehicles.

Wieting [29] experimentally investigated the effect of sweepback on the pressure and thermal loads of the shock interaction. The results indicated that the peak pressure and heat flux on the surface reduced with increasing sweepback. Wieting [30] and Hsu and Parpia [31] studied the effect of dual impinging oblique shocks interacting with a bow shock. In their work, concomitant jets or dual type IV jets were observed, and the shock-interference heating was decreased by the interaction of the noncoalesced dual impinging oblique shocks with a bow shock. Thus, we conjecture that the interaction of the noncoalesced isentropic compression waves (ICWs) with a bow shock can also effectively reduce the peak pressure and heat flux because the isentropic compression waves can be deemed a series of weak shocks. This interaction usually occurs when the isentropic compression waves from the inlet compression surfaces are designed to converge on the cowl lip to optimize the performance of the inlet. The interaction is characterized by the planar isentropic compression waves interacting with a cylindrical leading edge oriented with its axis parallel to the plane of the isentropic compression waves. Nevertheless, few studies have been conducted to analyze this type of interaction and the corresponding pressure and thermal loads. From a practical point of view, it is desirable for inlet designers to be able to predict this interaction and its effect. From an academic point of view, it is desirable to understand this specific kind of interaction as a fluid flow phenomenon to achieve the control of thermomechanics loading. The objective of this paper is to elucidate with numerical simulation the flow patterns, pressure, and thermal loads from the interaction of isentropic compression waves with a curved bow shock in front of a cylindrical body.

\section{Description of the Interaction}

The interaction of isentropic compression waves with a bow shock can be classified into two categories. One is the interference of the noncoalesced isentropic compression waves with a bow shock (sketched in Fig. 1a). For this category, we still know nothing about its interference patterns, interference pressure, and thermal loads. The other is the interference of the coalesced isentropic compression waves with a bow shock (sketched in Fig. 1b) where a straight coalesced shock wave forms before intersecting the curved bow shock. This kind of interference is equivalent to an oblique shock interacting with a bow shock, in which there may exist six shockinterference patterns, as defined by Edney in [3].

From characteristics of the two categories, the flow patterns as well as the pressure and thermal loads resulting from isentropic compression waves interfering with a bow shock depend on not only the location of the intersecting point relative to the curved bow shock but also the size of the region in which the isentropic compression waves intersects the bow shock. These geometric influencing factors can be uniquely denoted by the position of the coalesced point relative to the blunt body center, but this technique is confronted with the difficulty to distinguish whether the isentropic compression waves coalesce before intersecting the bow shock or not. For this reason, a relative intersection region size (RIRS), i.e., the ratio of a specific length of the isentropic compression waves intersecting the bow shock to the strong portion length of the undisturbed flow bow shock, is introduced to visually reflect the convergence degree of the isentropic compression waves. Here the specific intersection length is defined as the vertical height $\left(L_{S^{\prime} E^{\prime}}\right.$ in Fig. 2a) of the intersection region under the condition that the angle bisector (the dash-dotted line CV in Fig. $2 \mathrm{a}$ ) of $\angle S^{\prime} \mathrm{CE}^{\prime}$ passes through the vertex point $\mathrm{P}$ of the undisturbed flow bow shock. $S^{\prime}, E^{\prime}$ denotes the point of the starting

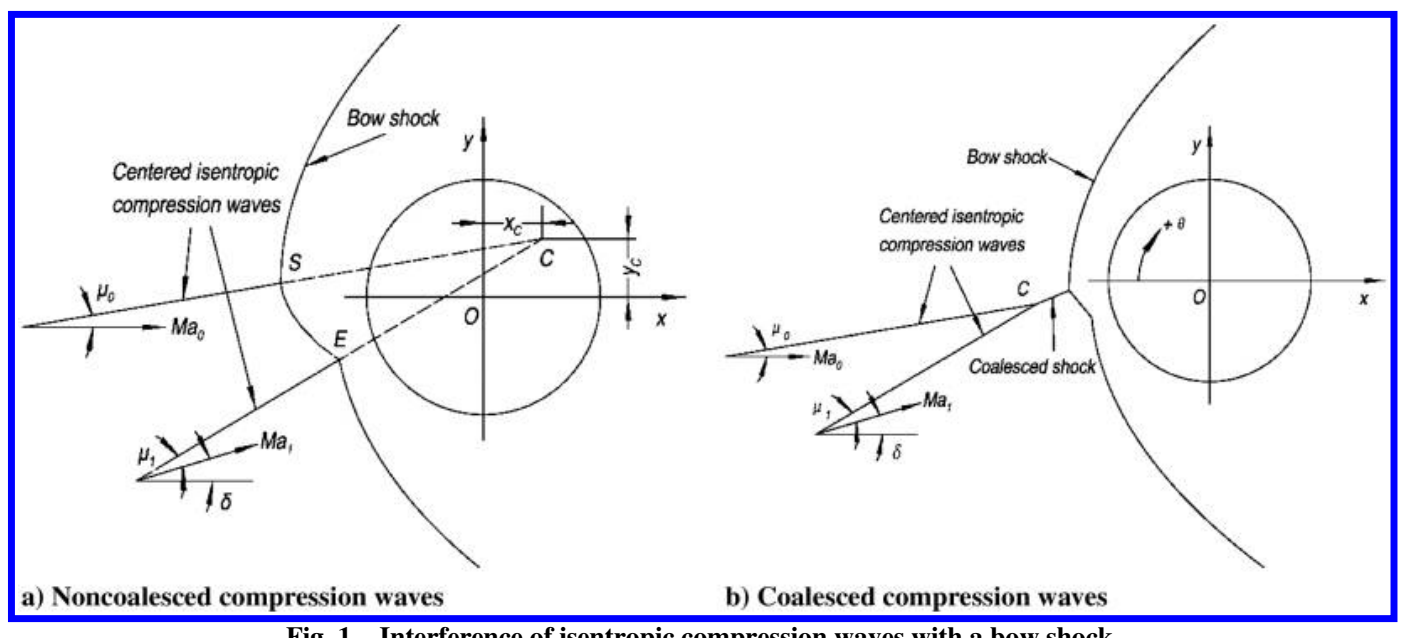

Fig. 1 Interference of isentropic compression waves with a bow shock. 


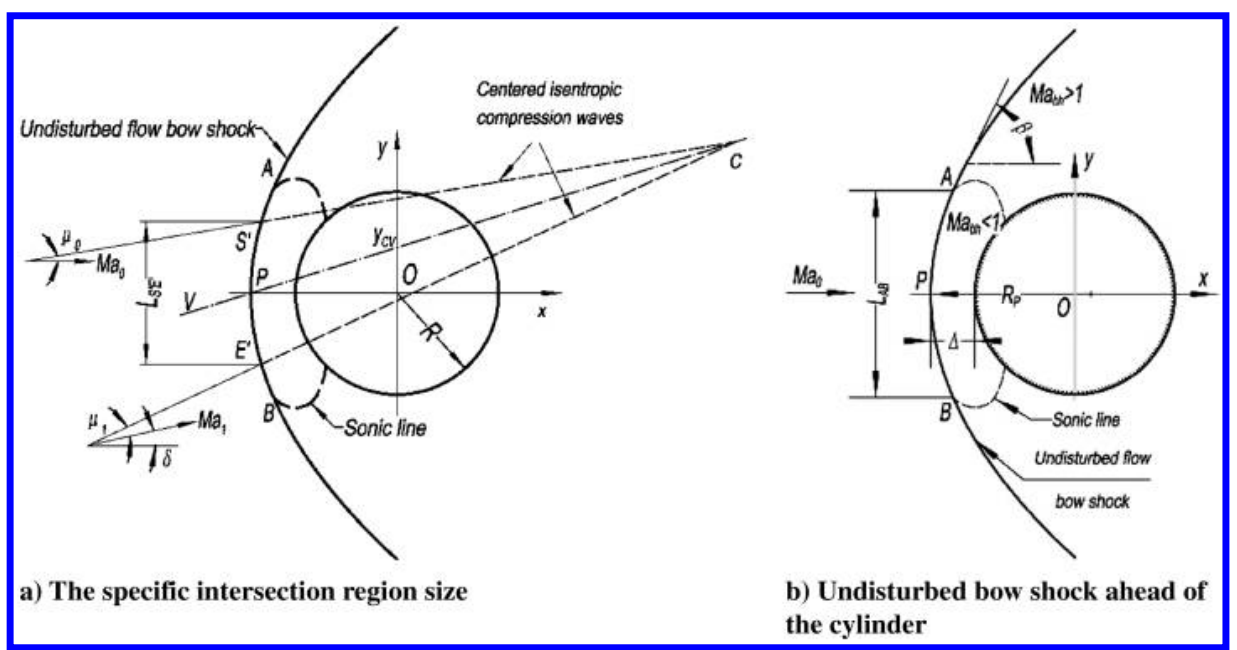

Fig. 2 Definition of the relative ideal intersection region size.

line $\mathrm{CS}^{\prime}$ and the ending line $\mathrm{CE}^{\prime}$ of the isentropic compression waves intersecting the undisturbed flow bow shock, respectively. The strong portion length of the undisturbed flow bow shock is denoted by the vertical distance ( $L_{A B}$ in Fig. 2 b) between the upper sonic point $\mathrm{A}$ and the lower one B. Correspondingly, RIRS $=L_{S^{\prime} E^{\prime}} / L_{A B}$. We view the undisturbed flow detached bow shock as a hyperbola expressed by the Billig relationship [Eq. (1)], which is given next [32]:

$$
\begin{aligned}
x & =R_{P} \cot ^{2} \mu_{0}\left(\left(1+\frac{y^{2} \tan ^{2} \mu_{0}}{R_{P}^{2}}\right)^{0.5}-1\right)-(R+\Delta) \\
R_{p} / R & =1.386 \exp \left(1.8 /\left(M a_{0}-1\right)^{0.75}\right)
\end{aligned}
$$

where

$$
\mu_{0}=\arcsin \left(\frac{1}{M a_{0}}\right) \quad \Delta / R=0.386 \exp \left(4.67 / M a_{0}^{2}\right)
$$

Based on the bow-shock shape defined by Eq. (1), the Mach number behind the undisturbed bow shock is obtained using the shock relationship. Accordingly, the vertical coordinate of the sonic points A and B and $L_{A B}$ are obtained. $L_{S^{\prime} E^{\prime}}$ is obtained through solving the vertical coordinates of the intersection points $S^{\prime}$ and $E^{\prime}$, which are determined by the predefined position of the coalesced point $\mathrm{C}$ relative to the cylinder center under the given conditions of $M a_{0}, R$, and $\delta$. From the definition of RIRS, it is found that the first category shown in Fig. 1a corresponds to RIRS $>0$, whereas the second category shown in Fig. $1 \mathrm{~b}$ corresponds to RIRS $=0$. Another geometric parameter characterizing the vertically intersecting position is denoted by $y_{\mathrm{CV}} / R$, where $y_{C V}$ is the vertical coordinate of the point at which the dash-dotted line CV intersects the $y$ axis, as shown in Fig. 2a. Under the given conditions of $M a_{0}, R$, and $\delta$, the two parameters RIRS and $y_{C V} / R$ can completely determine the position of the isentropic compression waves relative to the bow shock.

In this paper, a computational model is employed to investigate the characteristics of isentropic compression waves interfering with a bow shock in front of a blunt body, as shown in Fig. 3. The impinging isentropic compression waves from an isentropic surface are approaching from the lower-left quadrant of the flowfield, with the flow approaching from the left. The isentropic surface is a streamline solved with the aid of the $\mathrm{P}-\mathrm{M}$ relationship according to the approaching Mach number $M a_{0}$ and the flow deflection angle $\delta$ across isentropic compression waves. The vertical distance $H_{C}$ between the coalesced point $C$ and the starting point of the isentropic compression waves is controlled to avoid the impingement of the distorted shock on the ramp wall. The blunt body is represented by a cylinder with the radius equal to $3 \mathrm{~mm}$. The relative position between the cylinder and the coalesced point of the isentropic compression wave, characterized by RIRS and $y_{C V} / R$, is varied to study the interference patterns, pressure, and heat-transfer rate.

\section{Numerical Methods and Validation}

\section{A. Numerical Algorithm}

The full Navier-Stokes equations for two-dimensional laminar flow are numerically solved by the finite-volume method. The numerical flux through each cell face is evaluated using a secondorder total variation diminishing scheme based on an approximate Riemann solver named Harten-Lax-van Leer contact (HLLC). The HLLC scheme can resolve the isolated shock and slip line exactly while remaining positively conservative [33-35]. The minmod limiter is used to suppress spurious oscillations near the discontinuities while high-order accuracy is retained away from the jumps. A second-order fully implicit scheme is employed to discretize the time terms. In addition, the methods of multigrid and dual time step are used to accelerate the convergence.

\section{B. Validation}

A case study is conducted to guarantee the credibility of the present numerical results. An interaction of a straight oblique shock with a bow shock in front of a cylindrical body is selected to verify the flowpattern-capture ability and the heat-flux accuracy of the solver code. The simulation conditions, taken from the experimental run 21 in [12], are listed as follows: the freestream Mach number $M a_{0}=8.03$; static pressure $P_{0}=868.739 \mathrm{~Pa}$; static temperature $T_{0}=122.11 \mathrm{~K}$;

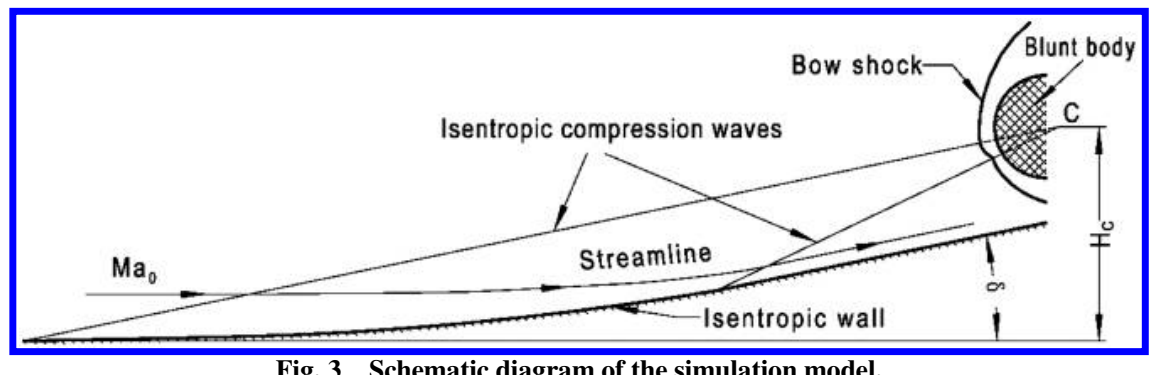

Fig. 3 Schematic diagram of the simulation model. 


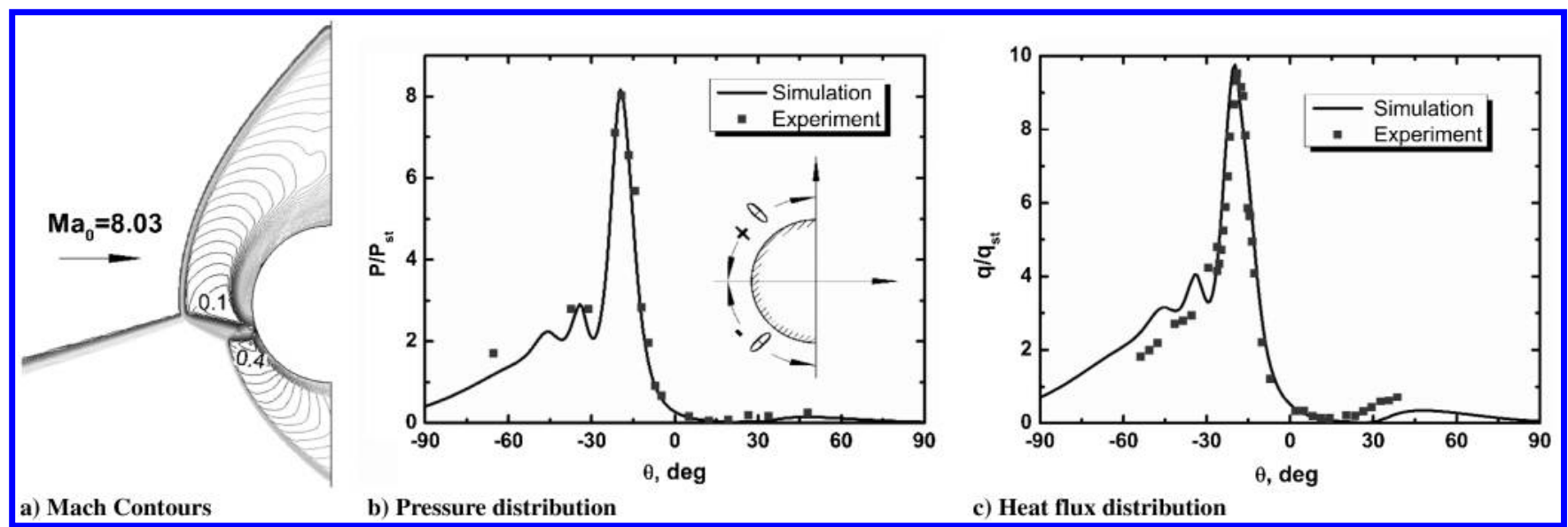

Fig. 4 Comparison between CFD and experimental results for an Edney IV shock pattern.

Reynolds number based on the radius of the cylinder, $R e_{R}=2.58 \times$ $10^{5}$; the impinging oblique shock angle $\beta=18.1 \mathrm{deg}$; and wall temperature $T_{w}=294.44 \mathrm{~K}$. An Edney type IV pattern is obtained, as shown in Fig. 4a, and the resultant pressure and heat flux distributions along the angular position of the cylindrical wall $\theta$ are depicted in Figs. $4 \mathrm{~b}$ and $4 \mathrm{c}$ together with experimental results [12]. Figure $4 \mathrm{a}$ shows Mach contours from 0 to 8 in increments of 0.1 and reveals that the main features of the type IV interaction are well resolved. The type IV pattern is composed of a three-shock $\lambda$ pattern, inverted $\lambda$ pattern, two strong vortex sheets, and a terminal shock. Note that a supersonic jet bounded by the two vortex sheets is embedded in the surrounding subsonic shock layer and ultimately terminates at the surface. During the jet development, there are successive isentropic-wave reflections from the two shear layers. At the base of the jet, a normal terminal shock is formed due to the extreme streamline curvature. The two shear layers turn rapidly upward and downward, respectively, with the strong pressure differential across the terminal shock. In Fig. $\underline{4 b}$, the static pressure $P$ on the surface is nondimensionalized with respect to the stagnationpoint pressure $\left(P_{\mathrm{st}}=73.15 \mathrm{kPa}\right.$, experimental data) without shock interaction in the same conditions. In Fig. $4 \mathrm{c}$, the heat flux $q$ on the surface is nondimensionalized with respect to the stagnation-point heat flux $\left(q_{\mathrm{st}}=0.7 \mathrm{MW} / \mathrm{m}^{2}\right.$, experimental data) without shock interaction in the same conditions. Figures $4 \mathrm{~b}$ and $4 \mathrm{c}$ indicate that simulation results are in good agreement with the experiment data.

\section{Computational Overview}

The calculations are performed on a single domain shown in Fig. 5a. According to the simulation conditions, the dimensions of the solution domain are varied to ensure that the distorted shock induced by the cylinder flows out at the downstream boundary and are listed in Fig. 5a. The lower boundary is mainly composed of the isentropic wall and the ramp wall, at which the slip conditions are adopted to guarantee that the isentropic compression waves are generated as designed. The symmetry conditions are employed at the lower boundary upstream the isentropic wall. At the upstream and upper boundary, the uniform freestream flow conditions are applied, whereas all variables were extrapolated at the downstream boundary. At the cylindrical wall, no-slip and isothermal conditions are used. The full domain is initialized by the uniform freestream flow conditions.

To enhance the resolution of the interference patterns, the $\mathrm{H}-\mathrm{C}$ grid shown in Fig. 5a is employed, where the $\mathrm{C}$ grid is used in a portion of the front face of the cylinder. Grid stretching is specially applied in the normal direction near the cylindrical and isentropic wall. A survey of the effects of grid refinement and the first mesh size $(\Delta n)$ normal to the cylindrical wall is conducted, and a sample is shown in Fig. $\underline{5 b}$. Based on the survey, the computations are ultimately performed using about the total number of 0.15 million, 0.25 million, and 0.3 million grids at the approaching Mach numbers of 6, 7, and 8, respectively. The corresponding first mesh size $\Delta n$ is $1,0.5$, and
$0.01 \mu \mathrm{m}$, respectively. Furthermore, the $\mathrm{C}$ grid includes $300 \times 200$ meshes in the radial (5R) and circumferential directions to well resolve the flow structure within the bow shock. To ensure the convergence, the heat flux at the frontal point of the cylinder is monitored. When it almost reaches constant or continuous repeatability, the solution is deemed converged.

\section{Numerical Results and Discussion}

A series of RIRS, $y_{C V} / R$, and Mach numbers are examined to investigate the flow characteristics as well as the pressure and thermal loads. The simulation is conducted for laminar flows with a perfect gas of constant specific ratio $(\gamma=1.4)$ and a standard atmospheric condition at $26 \mathrm{~km}$ altitude. The isentropic compression waves are produced by an isentropic surface sketched in Fig. 3 . The cylindrical surface is assumed to be the condition of an isothermal-constant

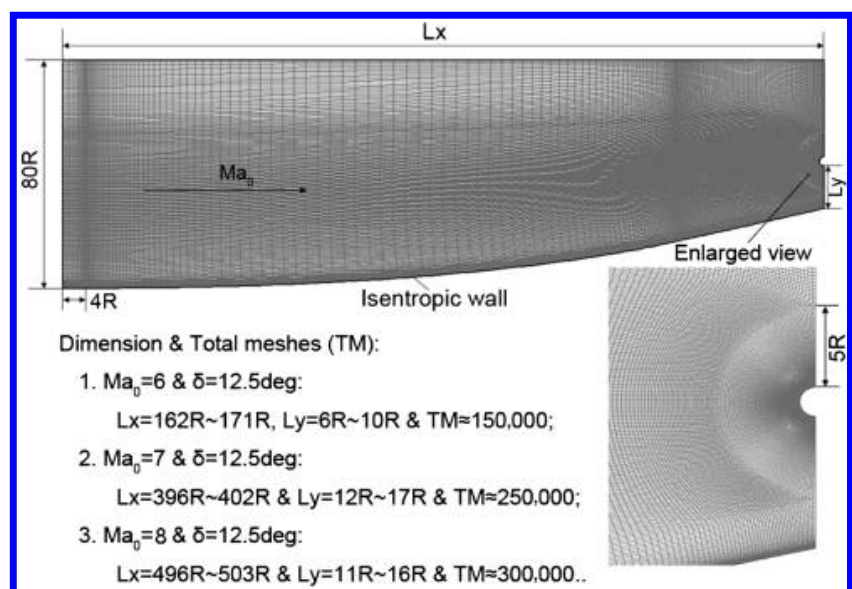

a) Computational domain and Grid
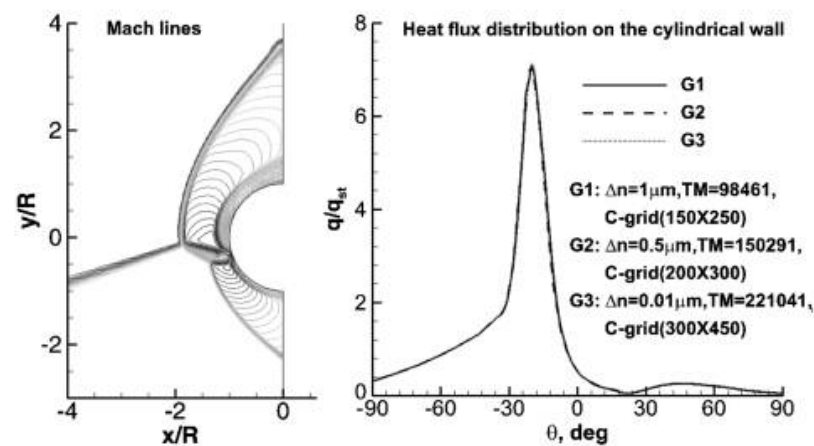

b) Grid refinement study where $M a_{0}=6, \mathrm{Re}_{R}=1.07 \times 10^{4} \delta=12.5 \mathrm{deg}$, RIRS $=0, y_{\mathrm{CV}} / R=0.09$

Fig. 5 Computational domain and grid-refinement study. 
temperature of $294.44 \mathrm{~K}\left(T_{w}=294.44 \mathrm{~K}\right)$. In the next discussion, the pressure $P$ on the cylindrical surface is nondimensionalized by the stagnation-point pressure corresponding to the freestream conditions, and the heat flux $q$ on the cylindrical surface is nondimensionalized with the stagnation-point heat flux corresponding to the freestream conditions.

\section{A. Effect of the Relative Intersection Region Size}

In this part, the effect of RIRS is examined by keeping the vertical location constant $\left(y_{C V} / R=0.45\right)$ under the conditions of $M a_{0}=6$, $\operatorname{Re}_{R}=1.07 \times 10^{4}$, and $\delta=12.5 \mathrm{deg}$. This vertical location corresponds to the isentropic compression waves intersecting the strong portion of the bow shock. Based on Eq. (1), we obtain the strong portion length of the undisturbed flow bow shock $L_{A B}=$ $6.19 \mathrm{~mm}$. Figures 6 and 7 show the flow patterns, the corresponding pressure, and heat-transfer distributions for a set of RIRS. As illustrated in Fig. 6a, an Edney IV interference pattern occurs when the coalesced isentropic compression waves interact with the strong portion of a bow shock. Correspondingly, the most serious heat transfer up to 12.1 times is created by the jet impinging on the surface. Amazingly, when RIRS is greater than 0 but small, a type IV interference pattern also occurs (shown in Fig. 6b), but a significant drop of $25 \%$ is observed in the heat transfer. Another noticeable difference between Figs. $6 \mathrm{a}$ and $6 \mathrm{~b}$ is the transmitted shock. The transmitted shock in Fig. 6a is straight, whereas the one in Fig. $6 \mathrm{~b}$ is composed of two segments: curved (SE) and approximately straight (ET). With a further increase of RIRS, a transonic jet emerges, as shown in Fig. 6c. When RIRS rises to 0.45, a subsonic jet is detected, as shown in Fig. 6d. The corresponding peak heat-transfer amplification reduces to 2.59 . As RIRS increases to 0.68 or larger, the interference pattern cannot be identified (e.g., as illustrated in Figs. 6e and $6 \mathrm{f}$ ). The corresponding peak heat transfer also drops remarkably.

These phenomena indicate that RIRS is a key factor governing the occurrence of the interference pattern. The Edney type IV interference occurs at a small RIRS, whereas the interference pattern disappears at a larger RIRS. These different flow patterns are attributed to a pressure jump across the isentropic compression waves in a limited flow space. To clearly elucidate the mechanism, a

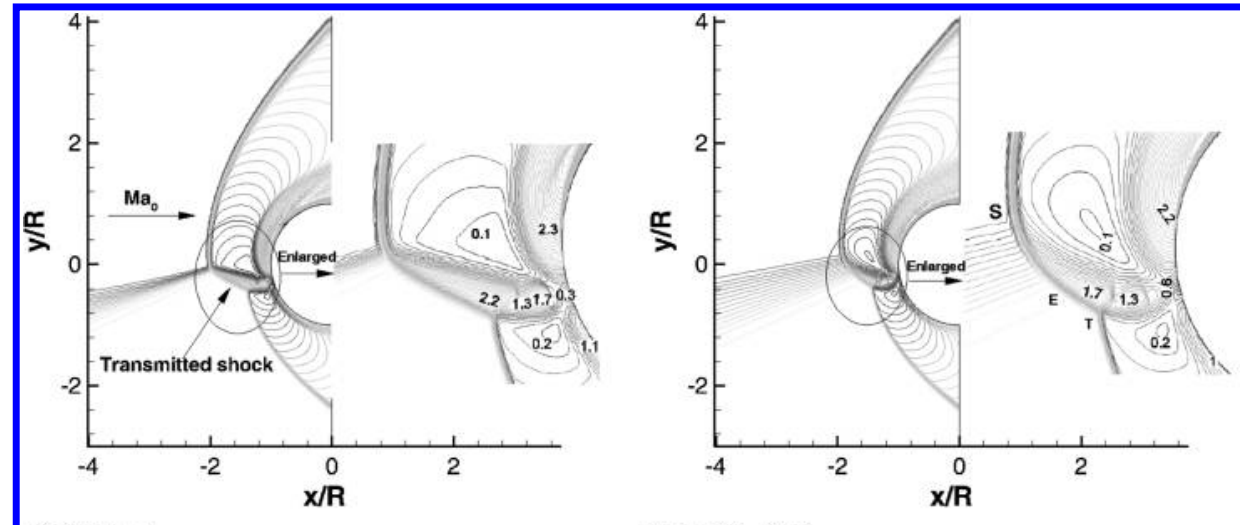

a) RIRS $=0$

b) RIRS $=0.21$
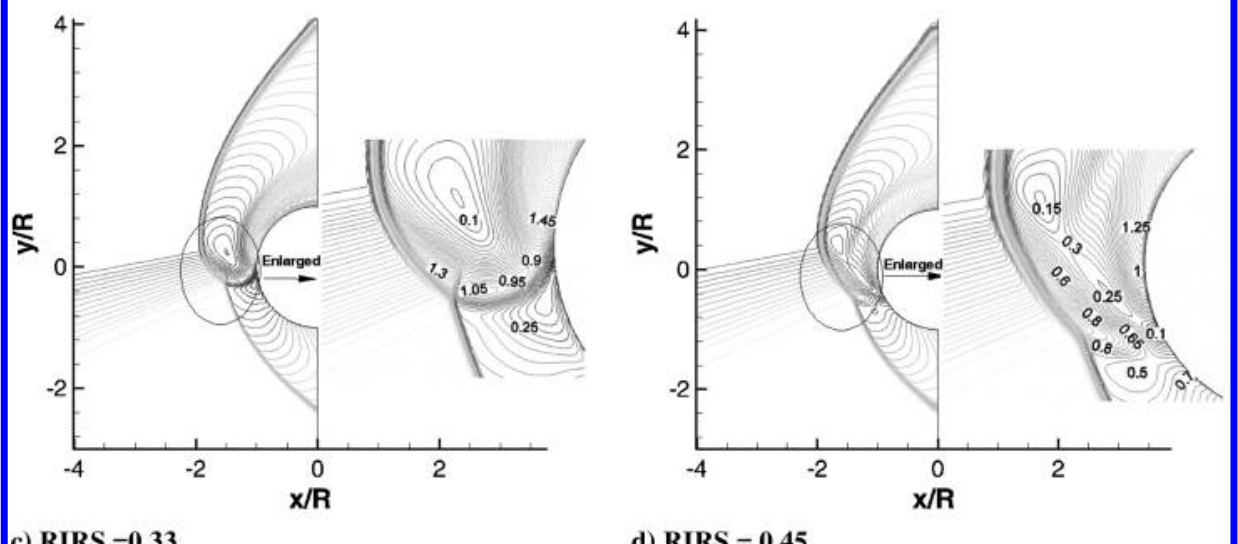

d) $\mathrm{RIRS}=0.45$
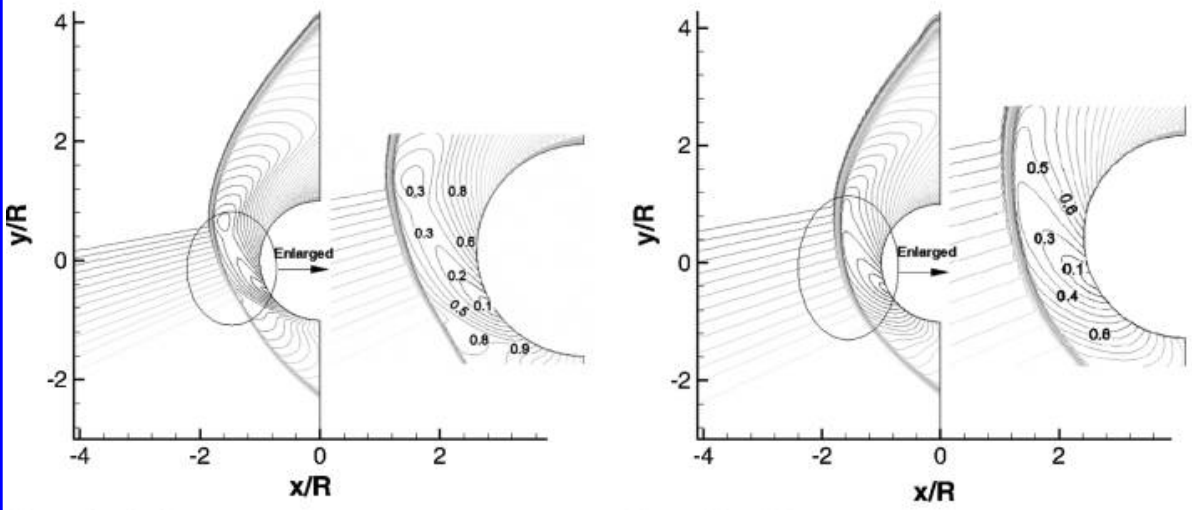

f) RIRS $=1.14$

Fig. 6 Mach contours for $y_{C V} / R=0.45, M a_{0}=6, \mathrm{Re}_{R}=1.07 \times 10^{4}$, and $\delta=12.5 \mathrm{deg}$. 


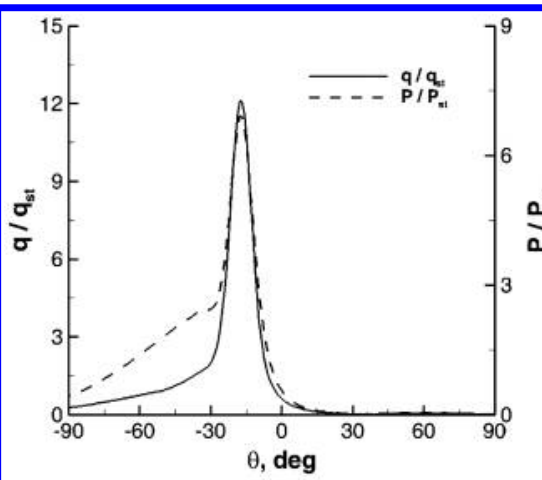

a) $\operatorname{RIRS}=\mathbf{0}$

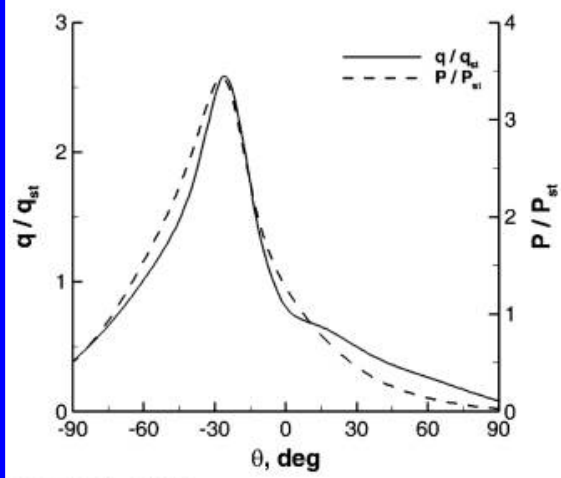

d) RIRS $=0.45$

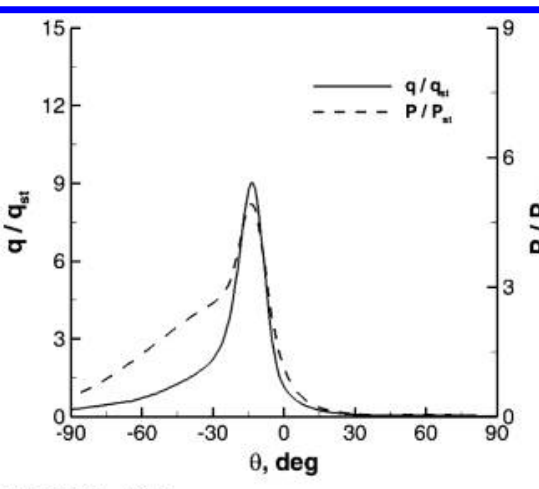

b) RIRS $=0.21$

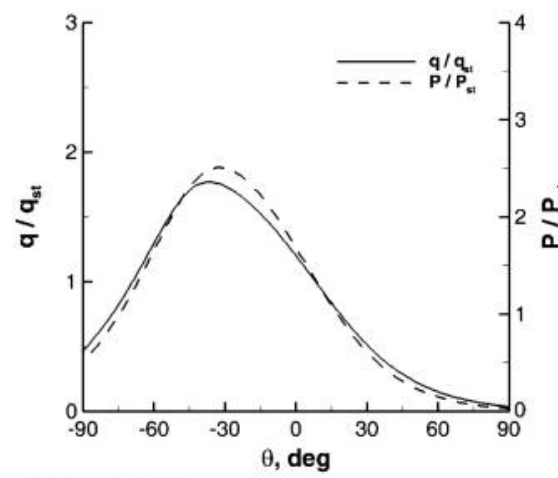

e) RIRS $=0.68$

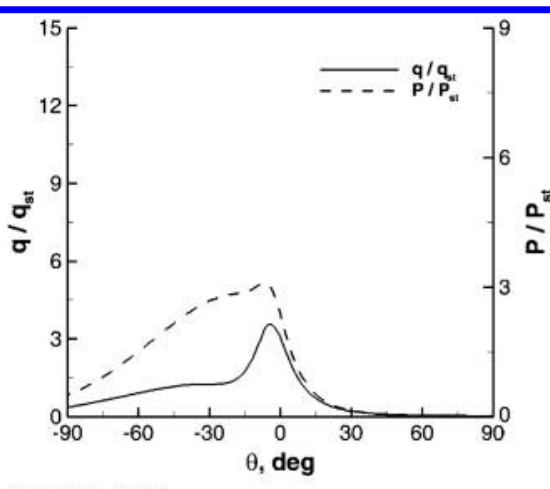

c) RIRS $=0.33$

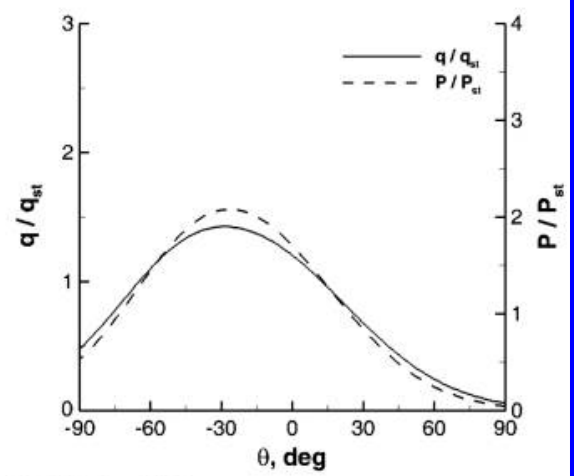

f) $\operatorname{RIRS}=1.14$

Fig. 7 Corresponding pressure and heat flux distributions on the cylindrical surface for $y_{C V} / R=0.45, M a_{0}=6, \mathrm{Re}_{R}=1.07 \times 10^{4}$, and $\delta=12.5$ deg.

physical flow pattern extracted from Fig. $6 \mathrm{~b}$ is shown in Fig. 8a. The transmitted shock ST is composed of two segments: curved $\overline{\mathrm{SE}}$ and straight ET. When the length of the curved part SE is much smaller than the total length of the transmitted shock ST, state 3 in Fig. 8a can be deemed as a uniform flow. Thus, the shock polar graphic of the solution to the oblique shock equations can be employed to solve the flow parameters of the states in Fig. $\underline{8}$. The corresponding map solution is illustrated in Fig. $8 \mathrm{~b}$ for $M \overline{a_{0}}=6, \delta_{1}=12.5 \mathrm{deg}$, and $\gamma=1.4$. State 1 is determined by the flow deflection angle across the isentropic compression waves $\delta_{1}=12.5 \mathrm{deg}$ and is on the curve ICW plotted using the P-M relationship. Accordingly, we can obtain the polar $M a_{1}$. The intersecting point of the polar $M a_{0}$ and the polar $M a_{1}$ is states 2 and 3 because $P_{3}=P_{2}$. The downstream conditions enforce a strong shock solution between states 0 and 2 . Similarly, states 4 and 5 are calculated by referring to the intersecting point of the polar $M a_{3}$ with the strong shock solution part of the polar $M a_{1}$. Ultimately, state 5 is obtained by the known parameters of $M a_{3}$ and $P_{5} / P_{3}$. The remaining states, states 6 and 7 , can be calculated using
$P-M$ relationships with $P_{6}=P_{2}, P_{7}=P_{4}$ and $P t_{6}=P t_{7}=P t_{5}$. It is indicated from Fig. $8 \mathrm{~b}$ that the Mach number controls the width, height, and slope of the symmetric heart-shaped shock polar. When the Mach number reduces, the polar becomes taller and higher. This tendency leads to a strong shock solution for states 2 and 4. As a result, the pressure match is bound to produce type III or IV interference (when the shear layer stemming from point $S$ is impossible to reattach to the wall, then type IV interference occurs).

With increasing RIRS, the curved part length of the transmitted shock increases, and the flow of state 3 becomes nonuniform but with approximately constant pressure $P_{3}$. As with the analysis of the map solution mentioned previously, the map solutions of the flow state behind the transmitted shock SE for $M a_{0}=6, \delta_{1}=12.5 \mathrm{deg}$, and $\gamma=1.4$ are a part of the polar $M a_{0}$, the portion between the critical point and point 3 in Fig. $8 \mathrm{~b}$. Here the solutions of the flow state in front of the shock SE are a portion of the ICW between the bottom point and point 1 , as illustrated in Fig. 8 b. The flow state 3 at point $S$ behind the transmitted shock corresponds to the critical point. It is

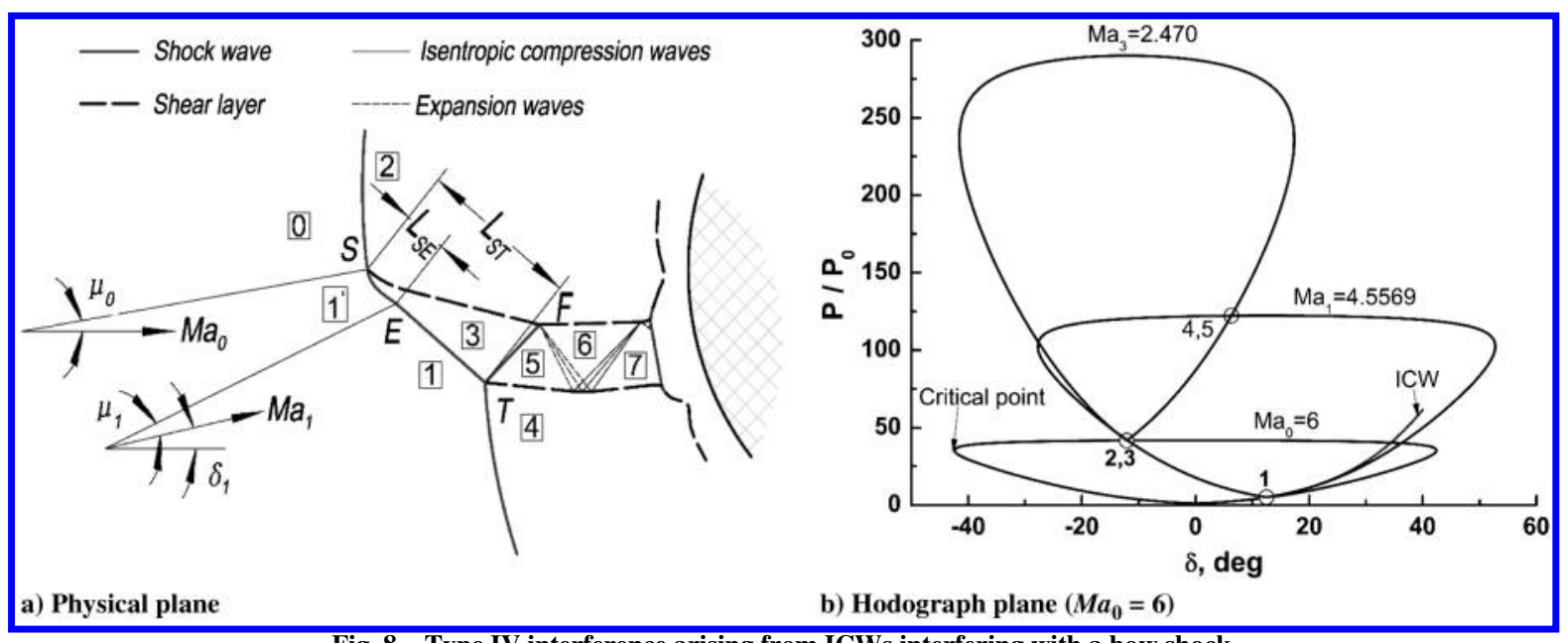

Fig. 8 Type IV interference arising from ICWs interfering with a bow shock. 
observed from the map that the flow deflection angle decreases gradually from the critical point to point 3 . This tendency implies that, from $\mathrm{S}$ to $\mathrm{E}$, the pressure ratio across the transmitted shock reduces, and the Mach number behind the transmitted shock increases under the conditions of $P_{3} \approx P_{2}$. As a result, the average Mach number of state 3 reduces with increasing RIRS at a constant $M a_{0}$ and $\delta_{1}$, and the terminal shock gradually moves upstream. When RIRS increases to some extent, a transonic jet is observed, as shown in Fig. 6c, where the flow in the jet decelerates to subsonic through the compression waves, rather than the terminal shock. When RIRS further increases to a transition point $\left(L_{\mathrm{SE}} \sim L_{\mathrm{ST}}\right)$, a subsonic jet is identified, e.g., Fig. 6d, where the flow behind the transmitted shock is completely subsonic but its Mach number is much greater than the neighbor zones'. With a further increase of RIRS to make the flow space large enough to match the pressure jump, the distinguishable interference pattern disappears (e.g., Figs. 6e and 6f). These phenomena of the interference pattern varying with RIRS $\overline{\text { reveals that }}$ the transmitted shock length is a critical scale of the intersection region size accounting for the mutual interaction, which determines the transition of the interference patterns. Furthermore, it is inferred that the interference patterns must disappear once the isentropic compression waves cover most of the front face of the cylinder (in other words, the intersection region fully occupies the strong portion of the detached bow shock, i.e., RIRS rises to the order of magnitude of 1), where the flow space is large enough to match pressure-jump.

\section{B. Effect of the Vertical Intersection Location}

At a constant RIRS, the characteristics of isentropic compression waves interacting with a bow shock depend on the vertical intersection location. To detail this effect, only some typical interference patterns are chosen next due to limited space, though a variety of model configurations have been tested for a series of fixed RIRS with $M a_{0}=6, \delta=12.5 \mathrm{deg}$.

Figure 9 shows Mach contours, pressure, and heat-transfer rate distributions for RIRS $=0.21$ at different vertical positions. The interference patterns defined by Edney [3] are identified as the intersection point moves from the lower to the upper parts of the bow shock. Edney type $\mathrm{II}^{+}$interference observed in Fig. 9a occurs when the isentropic compression waves cross beneath the lower sonic point. The peak heat-transfer rate is smaller than the freestream stagnation-point heat flux without interference due to the increasing detached distance of the bow shock. Type III interference observed in Fig. $9 \mathrm{~b}$ takes place when the compression waves cross around the lower sonic line. Type IV interference observed in Figs. $6 \mathrm{~b}$ and $9 \mathrm{c}$ is characterized by a supersonic jet embedded in the subsonic shock layer and produces the most severe heat-transfer rate. The angular position of the peak heat flux is located at the jet stagnation point. Type IVa interference illustrated in Fig. 9d is characterized by a supersonic jet grazing the surface. As the vertical intersection position moves along the $y$ axis for RIRS $=0.21$, a maximum heat flux occurs when the type IV supersonic jet impinges perpendicularly on the surface, just the same as the previous findings with regard to an oblique shock interacting with a bow shock.

Figure 10 shows Mach contours, pressure, and heat-transfer rate distributions for RIRS $=0.45$. In combination with Fig. $\underline{6 d}$, it is found that only part of the six Edney interference patterns occur as the vertical intersection position moves along the $y$ axis. Type $\mathrm{II}^{+}$and III interference patterns illustrated in Figs. 10a and 10b occur as usual, whereas type IV interference cannot be identified. Instead, a new pattern shown in Fig. 6d is observed where a subsonic jet is embedded in the shock layer. When the vertical intersection location moves further upward, the subsonic jet turns upward and flows away from the wall with the high pressure on the lower region, as shown in Figs. 10c and 10d.

Figure 11 shows Mach contours, pressure, and heat-transfer rate distributions for RIRS $=0.91$. Little difference is observed in the flow patterns when the vertical position moves from the lower to the upper parts of the bow shock. The shock wave in front of the cylinder is composed of three parts; the first part is a detached bow generated by the cylinder encountering with the freestream, the second is a curved shock generated by the cylinder being exposed to the isentropic compression waves, and the third is a detached shock generated by the cylinder encountering with the flow behind the isentropic compression waves. The heat-transfer rate distribution

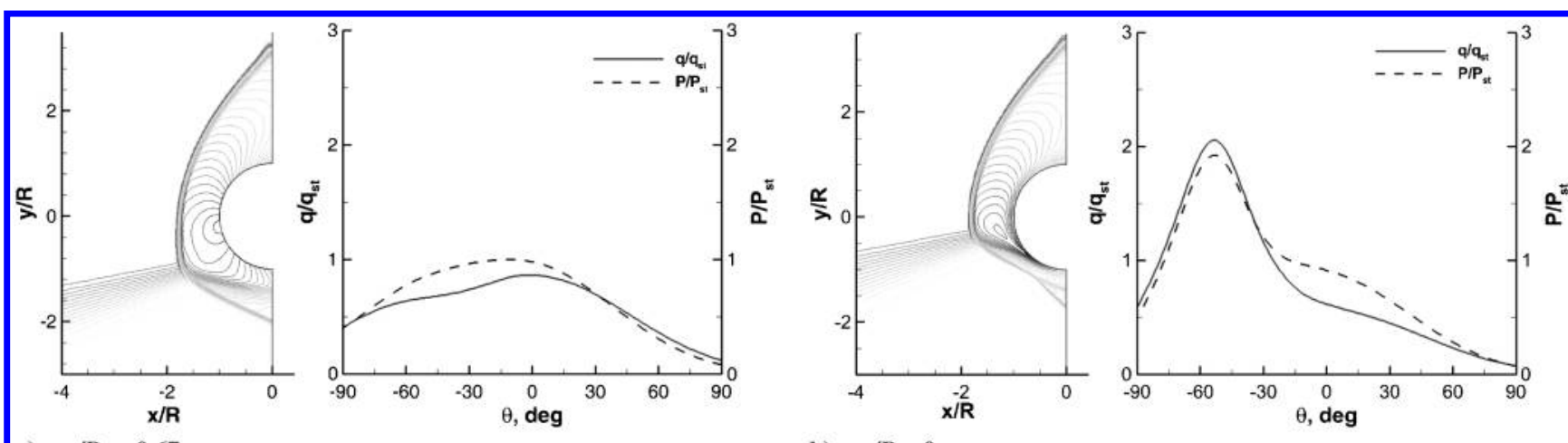

a) $y_{C V} / R=-0.67$
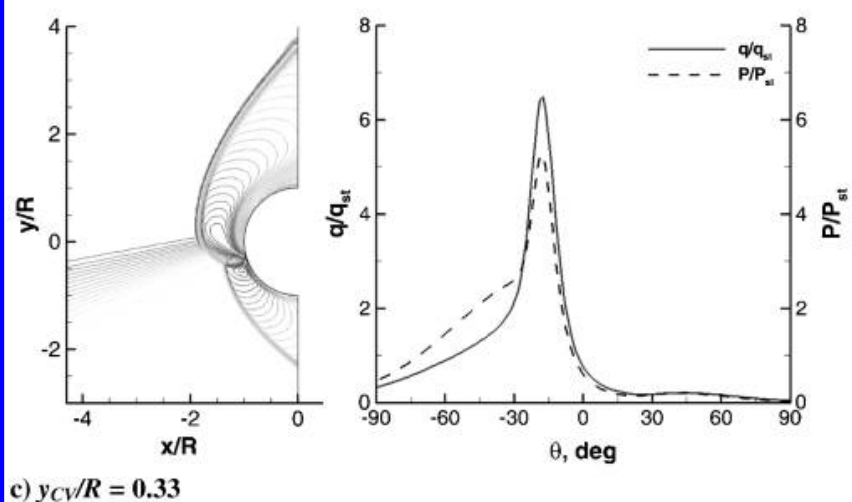

b) $y_{C V} / R=0$
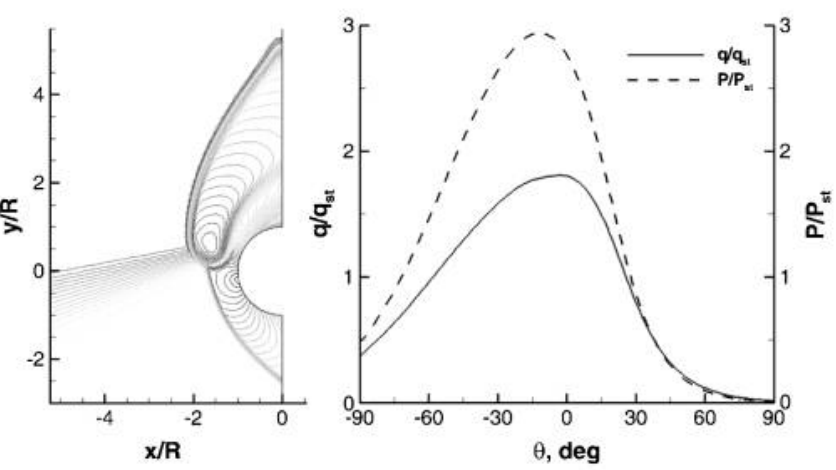

d) $y_{C V} / R=0.83$

Fig. 9 Mach contours, pressure, and heat flux distributions on the cylindrical surface, where $\mathrm{RIRS}=0.21, M a_{0}=6, \mathrm{Re}_{R}=1.07 \times 10^{4}$, and $\delta=12.5 \mathrm{deg}$. 


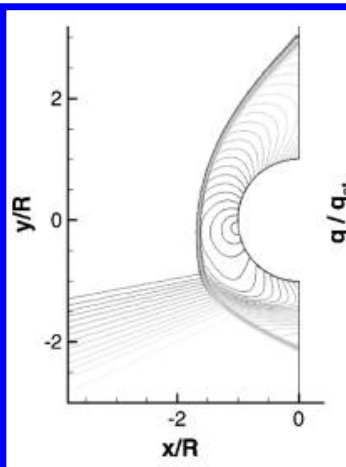

a) $y_{C V} / R=-0.85$

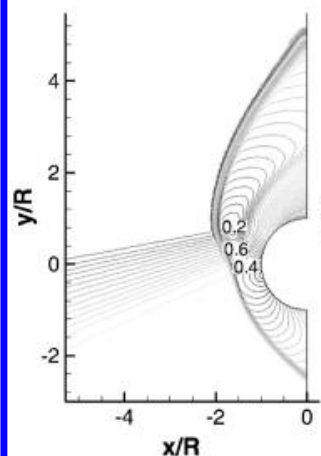

c) $y_{C V} / R=0.81$

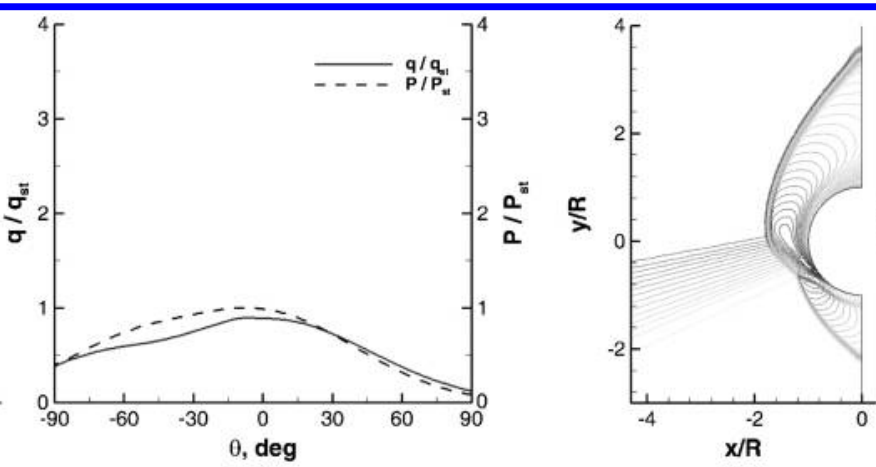

b) $y_{C V} / R=0.15$

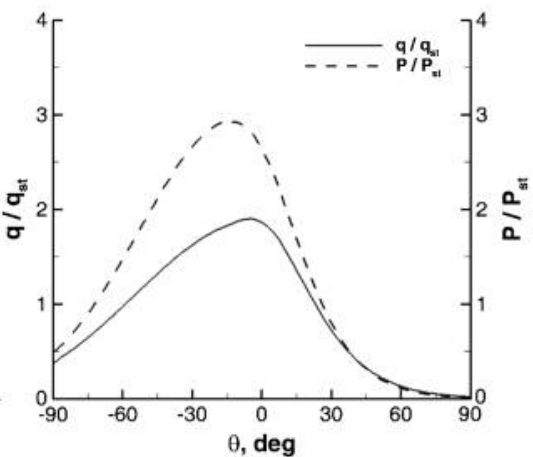

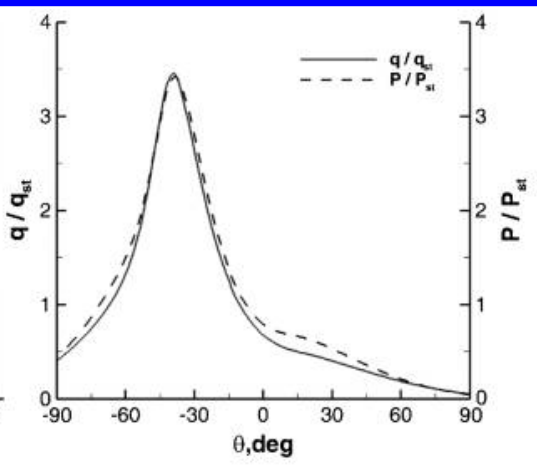

$\frac{a}{a}$

Fig. 10 Mach contours, pressure, and heat flux distributions on the cylindrical surface, where $\mathrm{RIRS}=0.45, M a_{0}=6, \mathrm{Re}_{R}=1.07 \times 10^{4}$, and $\delta=12.5$ deg.

also exhibits a similar shape, but the peak-heat-flux varies with the vertical intersection position. For $y_{C V} / R=-0.22,0.45,1.12$, and 1.78 , the peak heat flux amplification is $1.22,1.53,1.89$, and 1.68 , respectively. This tendency results from the effects of the distorted shock wave and the different flow conditions around the cylinder.
Referring to the results obtained in Sec. IV.A, we outline the overall effects of RIRS and $y_{C V} / R$ in Fig. 12. If the transonic jet is classified as a special Edney type IV interference, the plane of $y_{C V} / R$ RIRS can be divided into three zones. The first dividing line (RIRS in Fig. 12) corresponds to the boundary of the absence of the Edney

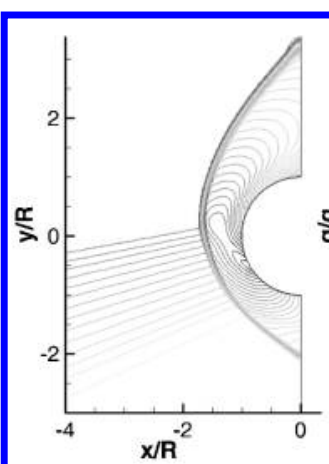

a) $y_{C V} / R=-0.22$

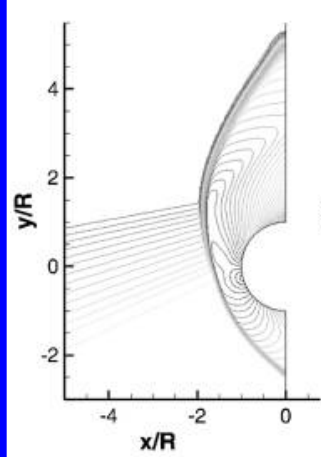

c) $y_{C V} / R=1.12$
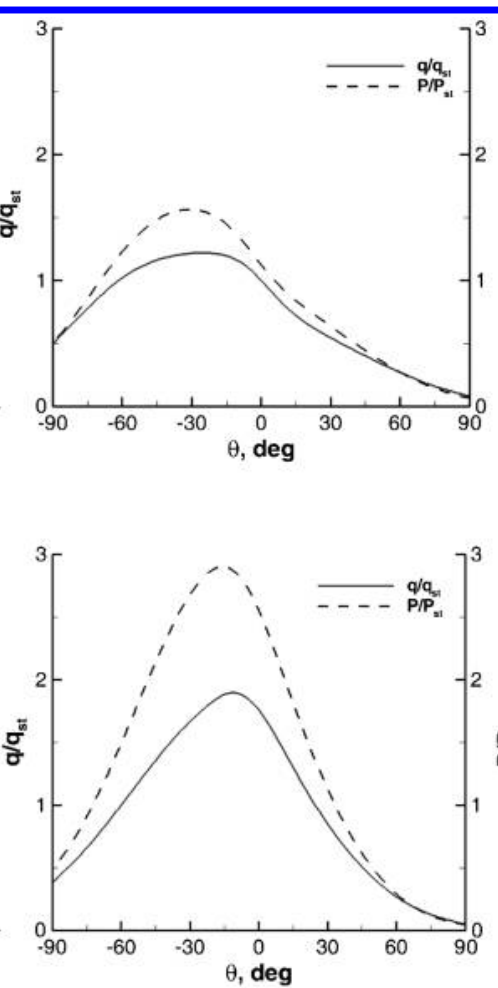
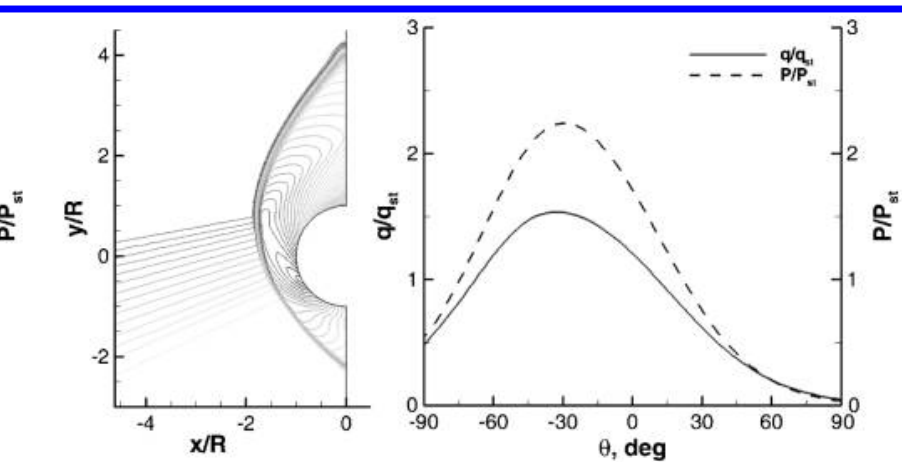

b) $y_{C V} / R=0.45$
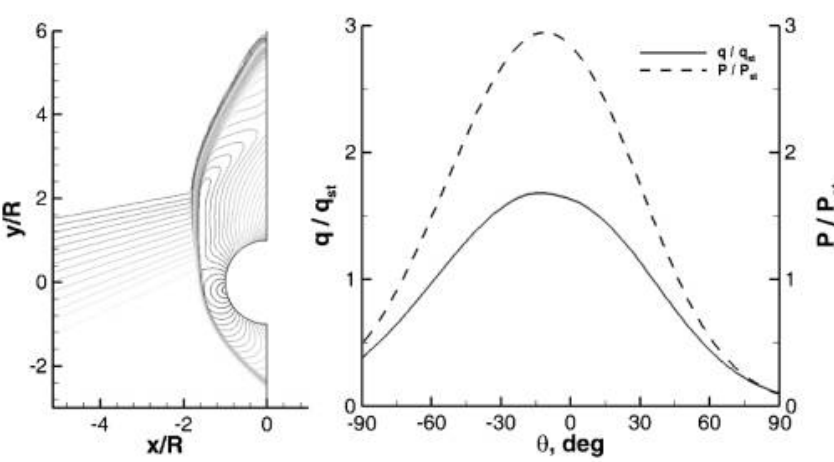

d) $y_{C V} / R=1.78$

Fig. 11 Mach contours, pressure, and heat flux distributions on the cylindrical surface for $\mathrm{RIRS}=0.91, M a_{0}=6, \mathrm{Re}_{R}=1.07 \times 10^{4}$, and $\delta=12.5$ deg. 
type IV pattern when the intersecting vertical location moves from the lower to the upper parts of the bow shock. The second dividing line $\left(\mathrm{RIRS}_{2}\right.$ in Fig. 12) corresponds to the boundary at which none of the Edney interference patterns occurs when the intersecting vertical location moves from the lower to the upper parts of the bow shock. When RIRS $<$ RIRS $_{1}$, the interference is similar to shock-on-shock interaction. The flow patterns, pressure, and thermal loads depend on the vertical intersection location. When RIRS $_{1}<$ RISRS $<$ RIRS $_{2}$, the interference is deemed as a special intermediate form, with the occurrence of a subsonic jet instead of a supersonic jet. The vertical intersection location also affects significantly the characteristics of this transition interference. When RISRS $>\mathrm{RIRS}_{2}$, the interference flow is simplified to a nonuniform flow with no distinguishable Edney interference patterns. The vertical intersection location has little impact on the flow patterns. From the physical mechanism of the formation of interference patterns, we conjecture that RIRS $_{1}$ corresponds to the intersection region size being approximately equal to the transmitted shock length in type IV interference, and RIRS corresponds to the intersection region size being close to the transmitted shock length in type III interference.

\section{Characteristics of the Peak Heat Flux}

The peak heat flux is the maximum heat-transfer rate on the cylinder surface at the fixed geometric position of the isentropic compression waves relative to the cylinder center. The peak heat-flux amplification is plotted in Fig. 13 versus the vertical intersection location for a set of RIRS. The amplification is defined as the ratio of the peak heat flux to the stagnation-point heat flux with the same Mach number and cylinder radius. In Fig. 13, the trend line is also plotted to specifically underline the effect of RIRS on the maximum heat flux. For a fixed RIRS, the peak heat flux amplification increases to reach a maximum before dropping back to a lower level as the vertical intersection location moves from the low to the upper parts of the bow shock. And this trend is particularly evident at small RIRS (e.g., RIRS $=0.21,0.47$ ) because the Edney interference patterns occur. In this situation, the maximum value is achieved when the

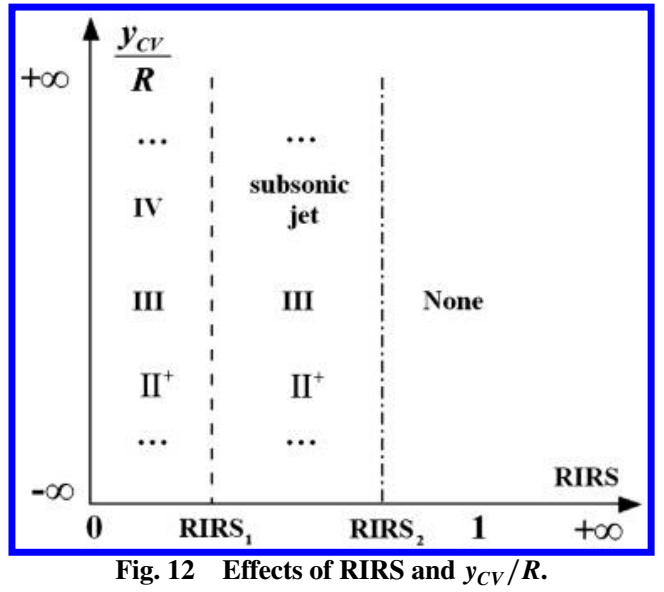

Table 1 Maximum heat transfer rates for a set of RIRS

\begin{tabular}{|c|c|c|c|c|c|c|}
\hline \multirow[b]{2}{*}{$\underline{\delta, \operatorname{deg}}$} & \multicolumn{2}{|c|}{$M a_{0}=6$} & \multicolumn{2}{|c|}{$M a_{0}=7$} & \multicolumn{2}{|c|}{$M a_{0}=8$} \\
\hline & RIRS & $q_{\max } / q_{\mathrm{st}}$ & RIRS & $q_{\max } / q_{\mathrm{st}}$ & RIRS & $q_{\max } / q_{\mathrm{st}}$ \\
\hline 12.5 & 0 & 12.12 & 0.22 & 8.52 & 0.23 & 9.21 \\
\hline 12.5 & 0.21 & 8.98 & 0.47 & 6.36 & 0.48 & 6.65 \\
\hline 12.5 & 0.45 & 5.32 & 0.72 & 3.53 & 0.84 & 2.87 \\
\hline 12.5 & 0.67 & 2.27 & 0.97 & 2.41 & 0.99 & 2.74 \\
\hline 12.5 & 0.91 & 1.89 & 1.21 & 2.15 & 1.14 & 2.34 \\
\hline 12.5 & 1.14 & 1.84 & -- & -- & - & -- \\
\hline 8 & 0.13 & 5.57 & $\begin{array}{c}M a_{0} \\
0.22\end{array}$ & 4.51 & 0.72 & 1.69 \\
\hline
\end{tabular}

supersonic jet impinges perpendicularly to the surface and is far larger than the stagnation-point heat flux without the interaction. It is also observed that the maximum amplification reduces sharply with increasing RIRS and reaches a very small value when RIRS is up to an order of magnitude of 1 . For example, the maximum amplification for RIRS $=0.91$ with Mach number 6, RIRS $=0.97$ with Mach number 7 , and RIRS $=0.99$ with Mach number 8 is $1.89,2.41$, and 2.74, respectively. These results are close to the stagnation-point heat flux under the flow conditions behind the isentropic compression waves using the Fay-Riddell formula [36] written in the following form:

$$
\begin{aligned}
q_{\mathrm{st}} & =0.567 \operatorname{Pr}^{-0.6}\left(\rho_{w} \mu_{w}\right)^{0.1}\left(\rho_{\mathrm{st}} \mu_{\mathrm{st}}\right)^{0.4} \\
& \times\left[2\left(P_{\mathrm{st}}-P_{0}\right)\right]^{0.25}\left(h_{\mathrm{st}}-h_{w}\right) / R^{0.5}
\end{aligned}
$$

The amplification predicted by Eq. (2) at Mach numbers 6, 7, and 8 is $1.77,1.95$, and 2.13 , respectively. These features reveal that the mutual interaction between an isentropic compression waves and a bow shock has little influence on the maximum thermal loads when RIRS goes up to 1 .

\section{Correlation of Maximum Heat Flux and Degree of Convergence}

As noted in Sec. IV.C, it is concluded that a maximum heat flux would occur as the vertical intersection location moves from the low to the upper parts of the bow shock. Table 1 lists the maximum heattransfer rates observed in the current work. To generalize our numerical results, we infer a dimensionless dependence of the maximum heat-transfer intensification $\left(\tilde{q}=q_{\max } / q_{\mathrm{st}}\right)$. Sanderson et al. [14] made a dimensionless analysis of the heat-transfer intensification from type IV shock interference. For a given gas, in the absence of the effects arising from gas dissociation and turbulent diffusion in the impinging jet, Sanderson regressed the dimensionless heat-transfer intensification $(\tilde{q})$ by the following expression:

$$
(\tilde{q}-1) \approx 9.0\left(M a_{0}-1\right)^{0.88} \delta^{0.78}
$$

For the interaction of the isentropic compression waves with a bow shock, RIRS is a key factor to determine the interference occurrence and the interference patterns. Hence, the maximum heat-transfer amplification $\left(\tilde{q}=q_{\max } / q_{\mathrm{st}}\right)$ in the current study must account for the effect of RIRS, and the dimensionless form turns out to be

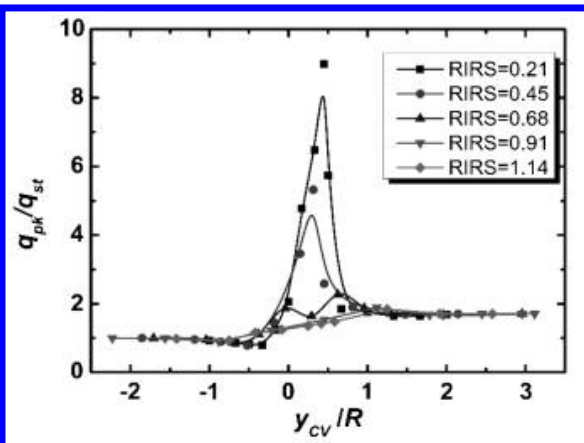

a) $M a_{0}=6, \mathrm{Re}_{R}=1.07 \times 10^{4}$

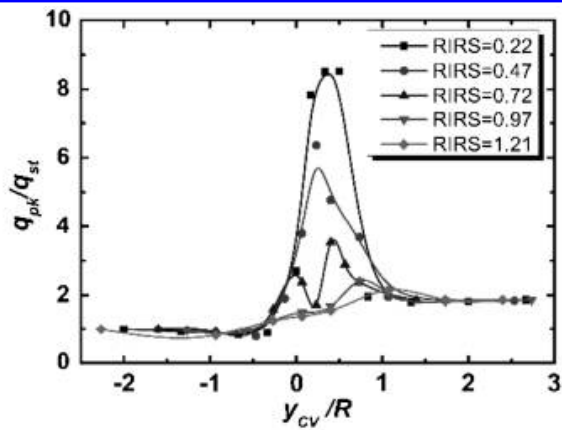

b) $M a_{0}=7, \mathrm{Re}_{R}=1.25 \times 10^{4}$

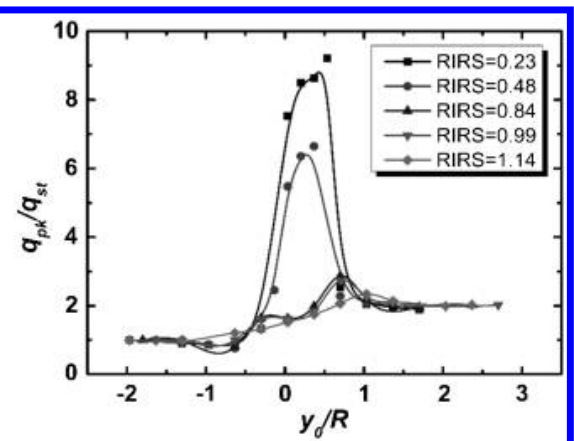

c) $M a_{0}=8, \mathrm{Re}_{R}=1.43 \times 10^{4}$

Fig. 13 Variation of peak heat flux with the vertical intersection location for a set of RIRS. 


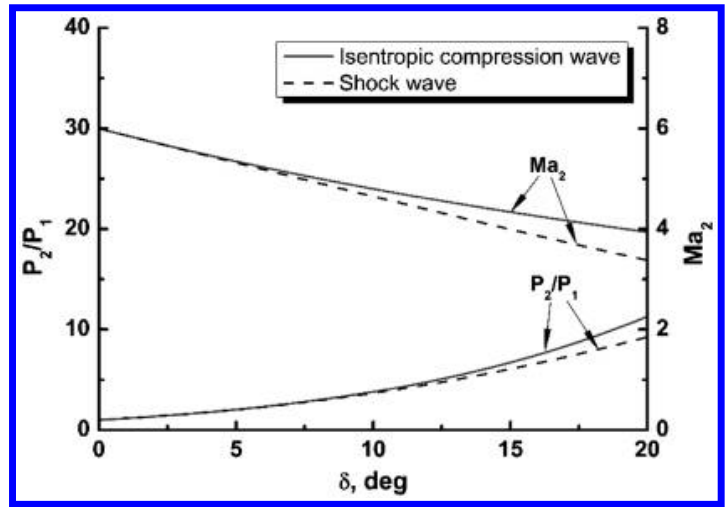

Fig. 14 Comparison of flow parameters cross isentropic compression waves and an oblique shock, where $M a_{0}=6$.

$$
\tilde{q} \approx f\left(M a_{0}, \delta, \text { RIRS }\right)
$$

On one hand, the interaction of the isentropic compression waves with a bow shock is equivalent to the shock-on-shock interaction with RIRS $=0$. Also, Fig. 14 shows that only a little difference exists in the flow parameters across the isentropic compression waves and a shock wave with the same approaching Mach number and flow deflection angle. These features indicate that the dimensionless expression involving RIRS should approach Eq. (3) in the limit RIRS $\rightarrow 0$. On the other hand, the maximum heat flux reduces with increasing RIRS, as demonstrated in Sec. IV.C. Based on the two features mentioned previously, we propose the following multiplicative form for the relationship between relevant parameters:

$$
(\tilde{q}-1) \approx \phi_{0}\left(M a_{0}-1\right)^{0.88} \delta^{0.78} \exp \left(-\phi_{1} \cdot \mathrm{RIRS}\right)
$$

Here, $\phi_{0}$ and $\phi_{1}$ are positive unknown constants. The previous experimental data regarding an oblique shock interfering with a bow shock are also used to regress the dimensionless relationship. These data are listed in Table 2, including the benchmark data of Edney [3] , the extensive Mach-Reynolds number scaling experiments of Wieting and Holden [12], and the nonequilibrium binary scaling data of Sanderson et al. [14]. Proceeding with the conventional method of logarithmic transformation and method of least squares for the unknown parameters in Eq. (5), we obtain

$$
(\tilde{q}-1) \approx 8.54\left(M a_{0}-1\right)^{0.88} \delta^{0.78} \exp (-2.31 \cdot \mathrm{RIRS})
$$

with a $96.7 \%$ correlation coefficient and with the parameters up to $>95 \%$ level of statistical significance. A little discrepancy exists in the constant coefficient between Eqs. (3) and (6) in the limit RIRS $\rightarrow 0$ due to the effects of statistical errors. The heat-transfer enhancement is plotted against the regression Eq. (6) in Fig. 15, which also presents 0 and $\pm 15 \%$ of the relative deviation between experimental or numerical data and those calculated by Eq. (6). It is noted that all the experimental and numerical data points almost fall within the residual $\pm 15 \%$ variability bands, which are attributed to

\begin{tabular}{|c|c|c|c|c|c|}
\hline Reference & $M a_{0}$ & $\delta, \operatorname{deg}$ & Geometry & Gas & $\tilde{q}$ \\
\hline Edney [3] & 4.6 & 5 & Sphere & Air & 6.5 \\
\hline Edney [3] & 4.6 & 10 & Sphere & Air & 7.5 \\
\hline Edney [3] & 4.6 & 15 & Sphere & Air & 10 \\
\hline Edney $[\overline{3}]$ & 7.0 & 5 & Sphere & Air & 8 \\
\hline Wieting and Holden [12] & 8.0 & 10 & Cylinder & Air & 13 \\
\hline Wieting and Holden $[\overline{12}]$ & 8.0 & 12.5 & Cylinder & Air & 18 \\
\hline Wieting and Holden $[\overline{12}]$ & 8.0 & 15 & Cylinder & Air & 25 \\
\hline Wieting and Holden $[\overline{12}]$ & 6.3 & 10 & Cylinder & Air & 11.5 \\
\hline Sanderson et al. [14] & 9.9 & 9.8 & Cylinder & $\mathrm{N}_{2}$ & 13.5 \\
\hline Sanderson et al. $[\overline{14}]$ & 6.3 & 7.7 & Cylinder & $\mathrm{N}_{2}$ & 7.5 \\
\hline Sanderson et al. [14] & 5.3 & 6.9 & Cylinder & $\mathrm{N}_{2}$ & 6 \\
\hline
\end{tabular}

Table 2 Heat flux intensification induced by type IV shock interference from previous studies

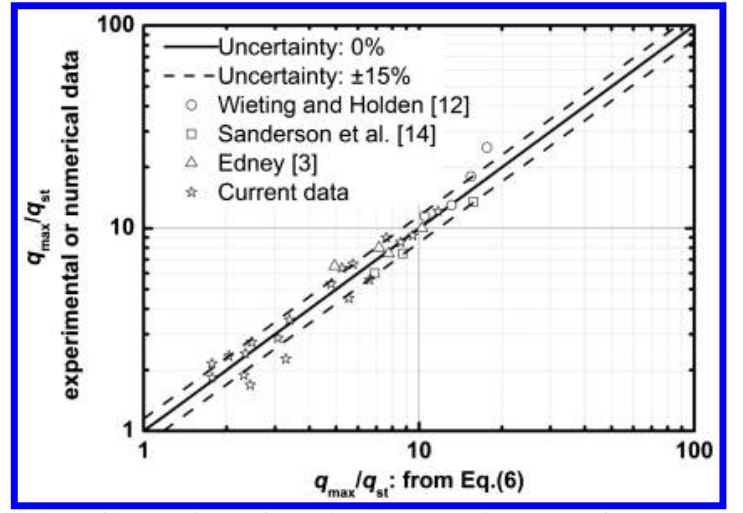

Fig. 15 Comparison of previous data against those from Eq. ( $\underline{6})$.

the effects of Reynolds number, the freestream total enthalpy, and so on. This feature implies that Eq. (6) can predict the maximum heating resulting from the interference of an oblique shock or isentropic compression waves with a bow shock, with fair accuracy. It can be concluded from Eq. (6) that the maximum thermal loads reduce exponentially with R IRS. This property indicates that the interference heating can be significantly minimized by dispersing the isentropic compression waves before intersecting the bow shock.

\section{Conclusions}

The interaction of isentropic compression waves with a bow shock in front of a cylindrical body is explored to achieve a shockinterference heating reduction by a modest modification of the wave system. The relative intersection region size (RIRS) is proposed to characterize this interaction and is defined as the ratio of a specific intersection region size to the strong portion length of the undisturbed flow bow shock. The effects of RIRS and the vertical intersection position on the flow patterns, pressure, and thermal loads from isentropic compression waves interacting with a bow shock are numerically discussed. Characteristics of the peak heat transfer are also investigated. Furthermore, a dimensionless dependence of the maximum heat-transfer intensification was inferred to generalize the current results. Some important conclusions are drawn.

Among the parameters involved, RIRS is a key factor governing the characteristics of the interaction. The interaction can be divided into three categories based on two critical RIRS. Edney interference patterns occur at a small RIRS and type IV interference pattern changes with an increase of RIRS. With increasing RIRS, flow Mach number downstream of the curved transmitted shock reduces, and the terminal shock gradually moves upstream until a transonic jet results. As RIRS increases to some value $\left(\mathrm{RIRS}_{1}\right.$ corresponds to the intersection region size that is approximately equal to the transmitted shock length in the case of Edney type IV interference), the type IV interference pattern transits to a special intermediate form where a subsonic jet occurs instead of a supersonic jet, coupled with a medium pressure and thermal loads. As RIRS exceeds another value $\left(\mathrm{RIRS}_{2}\right)$, none of the interference patterns takes place, which is associated with a lower pressure and thermal loads. Here, RIRS $_{2}$ corresponds to the intersection region size that is close to the transmitted shock length in the case of Edney type III interference.

A maximum heat flux occurs when the vertical intersection location moves from the lower sonic point to the upper sonic point of the bow shock at a constant RIRS. This maximum value reduces sharply with increasing RIRS. As RIRS is up to 1, the maximum pressure and thermal loads are similar to those of the stagnation point under the flow conditions behind the isentropic compression waves.

The dimensionless maximum heat-transfer enhancement is derived as a function of the approaching Mach number, the flow deflection angle, and RIRS. This formula can predict the maximum interference heating with fair accuracy. The maximum intensification drops exponentially with RIRS. The pressure and thermal loads can be minimized by a medium dispersion of isentropic compression waves. Furthermore, the database of the interference heating is provided for the design of practical vehicles. 


\section{Acknowledgments}

This work was supported by the National Natural Science Foundation of China (grants 90716014 and 91216115). The authors wish to thank all the members of our work group. The valuable and constructive suggestions of reviewers are greatly appreciated.

\section{References}

[1] Bertin, J. J., and Cummings, R. M., "Critical Hypersonic Aerothermodynamic Phenomena," Annual Review of Fluid Mechanics, Vol. 38, Jan. 2006, pp. 129-157. doi:10.1146/annurev.fluid.38.050304.092041

[2] Heppenheimer, T. A., "Facing the Heat Barrier: A History of Hypersonics," NASA SP-2007-4232, 2007.

[3] Edney, B. E., "Anomalous Heat Transfer and Pressure Distributions on Blunt Bodies at Hypersonic Speeds in the Presence of an Impinging Shock," Aeronautical Research Inst. of Sweden, FFA Rept. 115, Stockholm, Feb. 1968.

[4] Ryan, B. M., "Summary of the Aerothermodynamic Interference Literature," Naval Weapons Center TN-4061-160, China Lake, CA, April 1969.

[5] Korkegi, R. H., "Survey of Viscous Interactions Associated with High Mach Number Flight," AIAA Journal, Vol. 9, No. 5, 1971, pp. 771-784. doi: $10.2514 / 3.6275$

[6] Keyes, J. W., and Hains, F. D., "Analytical and Experimental Studies of Shock Interference Heating in Hypersonic Flow," NASA TN-D-71-39, May 1973.

[7] Watts, J. D., "Flight Experience with Shock Impingement and Interference Heating on the X-15-2 Research Airplane," NASA Ames Research Center TM-X-1669, Moffett Field, CA, Oct. 1968.

[8] Watts, J. D., and Olinger, F. V., "Heat-Transfer Effects of Surface Protuberances on the X-15 Airplane," NASA Flight Research Center TM-1566, Edwards AFB, CA, May 1968.

[9] Prabhu, R. K., "An Implementation of a Chemical and Thermal Nonequilibrium Flow Solver on Unstructured Meshes and Application to Blunt Bodies," NASA CR-194967, Aug. 1994.

[10] Dechaumphai, P., Thornton, E. A., and Wieting, A. R., "Flow-Thermal Structural Study of Aerodynamically Heated Leading Edges," Journal of Spacecraft and Rockets, Vol. 26, No. 4, 1989, pp. 201-209. doi: $10.2514 / 3.26055$

[11] Craig, R. R., and Ortwerth, P. J., "Experimental Study of Shock Impingement on a Blunt Leading Edge with Application of Hypersonic inlet Design," U.S. Air Force Aero Propulsion Lab. TR-71-10, April 1971.

[12] Wieting, A. R., and Holden, M., "Experimental Shock-Wave Interference Heating on a Cylinder at Mach 6 and 8," AIAA Journal, Vol. 27, No. 11, 1989, pp. 1557-1565. doi:10.2514/3.10301; also AIAA Paper 1987-1511, June 1987.

[13] Borovoy, V. Y., Chinilov, A. Y., Gusev, V. N., Struminskaya, I. V., Delery, J., and Chanetz, B., "Interference Between a Cylindrical Bow Shock and a Plane Oblique Shock," AIAA Journal, Vol. 35, No. 11, 1997, pp. 1721-1728. doi: $10.2514 / 2.41$

[14] Sanderson, S. R., Hornung, H. G., and Sturtevant, B., "The Influence of Non-Equilibrium Dissociation on the Flow Produced by Shock Impingement on a Blunt Body," Journal of Fluid Mechanics, Vol. 516, 2004, pp. 1-37. doi: $10.1017 /$ S0022112004000357

[15] Lind, C. A., "Effect of Geometry on the Unsteady-Type IV Shock Interaction," Journal of Aircraft, Vol. 34, No. 1, 1997, pp. 64-71. doi: $10.2514 / 2.2136$

[16] Berry, S. A., and Nowak, R. J., "Fin Leading-Edge Sweep Effect on Shock-Shock Interaction at Mach 6," Journal of Spacecraft and Rockets, Vol. 34, No. 4, 1997, pp. 416-425. doi: $10.2514 / 2.3247$

[17] Wang, W., Xie, L., and Guo, R., "Influence of Blunting Manner of the Lip Highlight of Hypersonic Inlet on the Aerothermodynamic Performance," 17th AIAA International Space Planes and Hypersonic Systems and Technologies Conference, AIAA Paper 2011-2306, April 2011.

[18] Lind, C. A., and Lewis, M. J., "Unsteady Characteristics of a Hypersonic Type IV Shock Interaction," Journal of Aircraft, Vol. 32, No. 6, 1995, pp. 1286-1293.

doi: $10.2514 / 3.46876$

[19] Lind, C. A., and Lewis, M. J., "Computational Analysis of the Unsteady Type IV Shock Interaction of Blunt Body Flows," Journal of Propulsion and Power, Vol. 12, No. 1, 1996, pp. 127-134.

doi: $10.2514 / 3.24000$

[20] Zhong, X., "Application of Essentially Nonoscillatory Schemes to Unsteady Hypersonic Shock-Shock Interference Heating Problems," AIAA Journal, Vol. 32, No. 8, 1994, pp. 1606-1616. doi: $10.2514 / 3.12150$

[21] Adelgren, R., Yan, H., Elliott, G., Knight, D., Beutner, T., and Zheltovodov, A., "Control of Edney IV Interaction by Pulsed Laser Energy Deposition,” AIAA Journal, Vol. 43, No. 2, 2005, pp. 256-269. doi: $10.2514 / 1.7036$

[22] Kogan, M. N., and Starodubtsev, M. A., "Reduction of Peak Heat Fluxes by Supplying Heat to the Free Stream," Fluid Dynamics, Vol. 38, No. 1, 2003, pp. 115-125. doi:10.1023/A:1023399331201

[23] Kandala, R., and Candler, G., "Numerical Studies of Laser-Induced Energy Deposition for Supersonic Flow Control," AIAA Journal, Vol. 42, No. 11, 2004, pp. 2266-2275. doi:10.2514/1.6817

[24] Trumble, K. A., and Candler, G. V., "Flow Control of Swept Fin Shock Interactions by Laser-Induced Plasmas," 44th AIAA Aerospace Sciences Meeting and Exhibit, AIAA Paper 2006-405, Jan. 2006.

[25] Yan, H., and Gaitonde, D., "Control of Edney IV Interaction of Energy Pulse," 44th AIAA Aerospace Sciences Meeting and Exhibit, AIAA Paper 2006-0562, Jan. 2006.

[26] Yan, H., and Gaitonde, D., "Effect of Energy Pulse on 3-D Edney IV Interaction," AIAA Journal, Vol. 46, No. 6, 2008, pp. 1424-1431. doi:10.2514/1.31530

[27] Gaitonde, D. V., and Miller, J. H., "Numerical Exploration of Shock Interaction Control with Plasma-Based Techniques," 34th AIAA Plasmadynamics and Lasers Conference, AIAA Paper 2003-3483, June 2003.

[28] Marley, C. D., and Riggins, D. W., "Numerical Study of Novel Drag Reduction Techniques for Hypersonic Blunt Bodies," AIAA Journal, Vol. 49, No. 9, 2011, pp. 1871-1882. doi:10.2514/1.J050681

[29] Wieting, A. R., "Shock Interference Heating in Scramjet Engines," 2nd International Aerospace Planes Conference, AIAA Paper 1990-5238, Oct. 1990.

[30] Wieting, A. R., "Multiple Shock-Shock Interference on a Cylindrical Leading Edge," AIAA Journal, Vol. 30, No. 8, 1992, pp. 2073-2079. doi:10.2514/3.11181; also AIAA Paper 1991-1800, June 1991.

[31] Hsu, K., and Parpia, I. H., "Simulation of Multiple Shock-Shock Interference Patterns on a Cylindrical Leading Edge," AIAA Journal, Vol. 34, No. 4, 1996, pp. 764-771. doi: $10.2514 / 3.13138$

[32] Billig, F. S., "Shock-Wave Shapes Around Spherical- and CylindricalNosed Bodies," Journal of Space and Rockets, Vol. 4, No. 6, 1967, pp. 822-823. doi:10.2514/3.28969

[33] Nagdewe, S. P., Shevare, G. R., and Kim, H. D., "Study on the Numerical Schemes for Hypersonic Flow Simulation," Shock Waves, Vol. 19, No. 5, 2009, pp. 433-442. doi:10.1007/s00193-009-0229-3

[34] Batten, P., Leschziner, M. A., and Goldberg, U. A., "Average-State Jacobians and Implicit Methods for Compressible Viscous and Turbulent Flows," Journal of Computational Physics, Vol. 137, No. 1, 1997, pp. 38-78. doi: $10.1006 / \mathrm{jcph} .1997 .5793$

[35] Toro, E. F., Riemann Solvers and Numerical Methods for Fluid Dynamics: A Practical Introduction, 3rd ed., Springer-Verlag, Berlin, 2009, Chap. 10.4.

[36] Fay, J. A., and Riddell, F. R., “Theory of Stagnation Point Heat Transfer in Dissociated Air," Journal of the Aeronautical Sciences, Vol. 25, No. 2 , 1958, pp. 73-85, 121. doi:10.2514/8.7517 